\title{
Computer Algebra System Maple: A New Software Library
}

\author{
Victor Aladjev \\ International Academy of Noosphere, Raadiku 13-75, 13817 Tallinn, Estonia \\ noosphere_academy@yahoo.com, academy60@hotmail.com
}

\begin{abstract}
The paper represents Maple library containing more than $\mathbf{4 0 0}$ procedures expanding possibilities of the Maple package of releases 6,7 and 8 . The library is structurally organized similarly to the main Maple library. The process of the library installing is simple enough as a result of which the above library will be logically linked with the main Maple library, supporting access to software located in it equally with standard Maple software. The demo library is delivered free of charge at request to addresses mentioned above.
\end{abstract}

\section{Introduction}

Computer Algebra (also known as Symbolic Computation or Computational Algebra) has found applications in many domains of science such as mathematics, physics, chemistry, computer science, engineering, technology, computational biology, education, etc. The computer algebra systems (CAS) such as Maple, Mathematica, Reduce, MuPAD, Magma, Derive, Macsyma, Axiom and others are becoming more and more popular and now they are valuable tools for teaching, research and industry.

In a series of our books the packages such as Reduce, Maple, MathCAD and Mathematica were considered [1-4]. Our experience of approval and use in different mathematical and physical appendices of four packages Reduce, Maple, Mathematica and MathCAD enables us to consider packages Maple and Mathematica as undoubted leaders (on the basis of a generalized index) among all listed modern means of computer algebra. Meanwhile, we prefer package Maple due to a number of important reasons which are not considered here.

Computer algebra becomes a rather powerful and useful tool for scientists and experts from various fields. However, manifold appendices demand significant numerical calculations combined with algebraic ones. With each new release, package Maple meets more and more requirements. Maple package has been widely used not only as means of solving mathematical problems. The use of the package enabled to revise approaches to teaching subjects related to mathematics in universities by defining in many cases the methods for subject development the use of PCs to solve mathematical problems of various purposes [1-4].

Researchers use Maple as an essential tool when solving problems related to their investigation. The package is ideal for formulating, solving, and exploring different mathematical models. Its symbolic manipulation facilities greatly extend the range of problems which can be solved by it. Educators in high schools, colleges, and universities have revitalized traditional curricula by introducing problems and exerci- 
ses which use Maple's interactive mathematics and physics. Students can concentrate on important concepts rather than on tedious algebraic manipulations. Finally, engineers and experts in industries use Maple as an efficient tool replacing many traditional resources such as reference books, calculators, spreadsheets and programming languages. These users easily solve a wide range of mathematical problems, creating projections and consolidating their computations into professional reports.

\section{A New Software Library for Maple}

Meanwhile, our operational experience during 1997-2002 with the Maple of releases 4, 5, 6, 7 and 8 has enabled us not only to estimate its advantages compared with other similar mathematical packages, but has also revealed a series of faults and shortcomings eliminated by us. Furthermore, the Maple does not support a series of important enough procedures of information processing, symbolic and numeric computing, including means of access to datafiles. In view of told, we in process of the Maple operation have developed a lot of effective enough software (procedures and modules), by a whole series of parameters expanding opportunities of the package. This software had been organized as a library which is structurally similar to the main Maple library, and is provided with detailed enough Help system analogous to Maple Help system.

The library elements had been written on built-in Maple language with usage in a series of cases of means of underlying operating system MS DOS. At that, for increase of their functional efficiency a lot of special receptions of programming (including non-standard ones) had been used. Among these receptions had been used so-called method of 'disk transits', which had been described in our books [1-4] enough in detail. In particular, the given method can be used the most advantageously in the problems, needing in a generating of Maple fragments executable in real-time. The essence of the given mechanism in the Maple environment is well illustrated in our last books [3, 4].

The presented software increases the range and efficiency of use of the package owing to the innovations in three basic directions: (1) elimination of a series of basic defects and shortcomings, (2) expanding of capabilities of a series of standard means, and (3) replenishment of the package by new means which increase the capabilities of its environment, including the means which improve the level of compatibility of releases 6,7 and 8 . The experience of use of the given software has confirmed its high operational characteristics at use of the Maple in numerous appendices.

As a matter of fact, the software has been developed as a self-installed Library which is analogous to the main Maple library. The given library is logically linked to the main Maple library, providing access to means contained in it similarly to standard means of the package. The given library supports the releases 6, 7 and 8; its installation provides essential increase of capabilities of the package Maple both for beginners and the advanced users, which use the package in various appendices.

The basic attention had been given to the additional means created in process of use of the package of releases 4-8 which by a series of parameters essentially expand opportunities of the package and facilitate work with it. The considerable attention is given to the means providing package compatibility of releases 6,7 and 8 . The 
experience of use of the given software has confirmed its high operational characteristics at use of the Maple in numerous appendices. The current version of the library contains means oriented upon the following kinds of processing:

1. Software of general destination

2. Software of operation with procedural and modular objects of the package Maple

3. Software of operation with numeric expressions

4. Software of operation with string and symbolic expressions

5. Software of operation with the lists, the sets and the tables

6. Software of support of data structures of a special type

7. Software of support of bit-by-bit processing of the information

8. The software expanding graphic possibilities of the package Maple

9. The software of expanding and improving the standard means of the Maple

10. Software of operation with datafiles and Maple-documents

10.1. Software of general destination

10.2. Software of operation with the TEXT datafiles

10.3. Software of operation with the BINARY datafiles

10.4. Software of operation with the Maple files

10.5. Special means of operation with datafiles

11. Software of operation with user libraries

12. Software for problems solving of mathematical analysis

13. Software for problems solving of linear algebra

13.1. Software of general destination

13.2. Software for operation with the rtable-objects

14. Software for support of problems of simple statistics

14.1. Software of problems solving of descriptive statistics

14.2. Software of problems solving of regression analysis

14.3. Software for a testing of statistical hypotheses

14.4. Elements of the analysis of time (dynamic) series

Basic innovations of the above software can be briefly characterized in the following way. Above all, the most general procedures of operation with Maple package are intended for receiving information such as number of the current Maple release, full paths to its main subdirectories, current version of an underlying system, information about installed Maple-releases, etc. (group 1). Similar means are absent among the standard Maple software, however they appear useful enough at more advanced operation with the Maple.

The next group of means expands the possibilities of the Maple package at operation with procedures and modules. These means support kinds of processing such as converting of modules into procedures; testing of presence in files of incorrect modules; check of parameters of procedures and modules; check of activity (availability for direct use) of a procedure or module; check of type of a modular table; a converting of files of input Maple-format containing modules; converting of a module of the second type into the first; converting of a file of input Maple-format into file of internal Maple-format, and vice versa, etc. (group 2). The represented means provide a lot of the manifold useful operations with the procedural and modular objects of the Maple. They are used enough widely at advanced programming of various problems in the Maple environment. 
Means of the third group expand the possibilities of the package at operation with numeric expressions. In particular, these means provide useful procedures such as converting of a floating-point number into a symbolic customary format, special converting of integers into lists, and vice versa, converting of an integer into symbolic format with delimiter, converting of number represented by a format $\{$ string, symbol $\}$ into fraction, etc.

The next means (group 4) expand the possibilities of the package at operation with expressions of type $\{$ string, symbol $\}$. These means provide a series of useful procedures such as: special kinds of converting; comparison of strings or/and symbols; case sensitive pattern searching; exhaustive substitutions into strings or symbols; inversion of symbols, a strings or lists; reducing of multiplicity of entries of a symbol into a string; identification of entries of special symbols into a string; and others. In a series of cases these means simplify operation with objects of type $\{$ string, symbol $\}$.

The fifth group represents the software which expands the possibilities of the Maple at operation with objects of types $\{$ list, set, table $\}$. The list structures play an extremely important role, defining the ordered sequences of elements. Since the sixth release, a possibility of substantial expanding of operations with the list structures arises. As an example having the interesting practical appendices, we consider definition of algebra on a set of all lists having the same length. Algebraic operations the corresponding procedures provide. A series of procedures of the group supports useful kinds of processing such as: special converting of lists into sets, and vice versa; operation with rarefied lists; dynamic assignment of values to elements of a list or a set; evaluation of indices of a table over its entry; representation of a special type of the tables; special kinds of exhaustive substitutions into a list or a set; a series of important kinds of sorting of nested lists, and also many others. The given means are useful enough at operation with objects of the above types.

The sixth group contains the expanded means of work with the data structures such as the stack, quеuе, priqueue, etc. Along with standard data structures of types $\{$ stack, queue, heap, priqueue\}, the new dirax-type maintained by module DIRAX had been defined. The module supports data structure of direct access of dirax type, which by a series of parameters essentially expands data structures of types \{stack, heap, queue . The data structures of the dirax type are founded on list organization, however allow to address itself immediately to its elements according to their addresses (numbers). Moreover, in contrast to structures of type $\{$ stack, queue $\}$, the dirax structure is precisely identified by ispd index; a reception, used in the module, allows easily to define precise classification of data structures of type $\{$ stack, quеие, heap, dirax $\}$. At the same time, the DIRAX module ensures the effective solution of problems of shared use of data structures of all above-mentioned types. Furthermore, means of testing of the above data structures types have been essentially precised, because the standard means have some inaccuracies.

In the seventh group the software which supports bit-by-bit information processing in environment of the Maple is represented. The package does not possess means of the similar type. The software offered by us is represented by six useful procedures such as: Bit, Bit1, xbyte, xbyte1, xNB and xpack. These procedures serve for bit-by-bit information processing, i.e. the user has a possibility to operate with strings or symbols on the level of separate bits composing them. 
The eighth group contains 60 procedures which expand and improve the standard means of the Maple of releases 6, 7 and 8. The package does not possess means of the similar type and their availability in a series of cases essentially expands possibilities of the package and simplifies operation with it. These means are used enough widely both at operation with Maple in interactive mode and at programming of various problems in its environment. In particular, the changes touched the assign procedure which is one of the reasons of incompatibility of releases 6,7 and 8 . The given problem has appeared rather essential at translating of software created in environment of the 6th release, into environment of the 7th and 8th release. The incompatibility problem of the last three releases of the Maple is considered in detail in our books [1-4]. The procedures represented in the group both eliminate the above problem of incompatibility of releases and enlarge the possibilities of the standard function assign by allowing, in particular, to assign a sequences of expressions to variables.

The subgroup of procedures Save, Save1, Save2 and Readl fulfills a much wider set of functions, than built-in functions 'save' and 'read', eliminating a series of essential shortcomings of the last. Along with that, the standard procedures 'convert" and 'type" have been essentially expanded and precised. In particular, a series of important types of data structures and expressions had been defined. So, new types for files, directories, paths to files, bytes have been determined, etc. The mechanism of types is very powerful facility of the Maple, therefore its further expanding is topical enough in my opinion. The other procedures of the ninth group also represent undoubted interest at programming of various problems in the Maple environment, both by simplifying the programming and by making it by more clear.

The ninth group contains the software expanding graphic possibilities of the Maple. The package does not possess means of the similar type. The software is represented by 12 useful procedures having undoubted applied interest.

Being the programming language in the package environment, oriented, first of all, onto symbolical calculations, the Maple-language has the relatively limited opportunities at operation with the data which are located in external memory. And in this plan the Maple-language essentially yields to traditional programming languages such as C, Cobol, Fortran, PL/1, Pascal, ADA, Basic, etc. At the same time, the Maple language, oriented, first of all, onto the solution of problems of mathematical character, gives a set of means for access to datafiles which can entirely satisfy a wide enough audience of users of physical and mathematical appendices of the package. The tenth group contains a lot of additional means of access to datafiles which essentially expand opportunities of the package in the given direction.

In particular, the subgroup from 50 procedures supports a series of important functions of access to datafiles, namely: operations with drives and volumes of external memory, operations with directories and files, renaming of directories and files, change of attributes of a directory and a file, check of properties of a directory or a file, and many other procedures, which support a multitude of useful functions of operation with elements of a PC's file system.

The next four subgroups contain means supporting a number of useful functions of work with datafiles of types TEXT and BINARY, with Maple files of other types, and a lot of means for special datafiles processing. In particular, means of these subgroups provide procedures such as: effective handling of situation "end of BINARY file", methods of organization of direct access to TEXT files, an extracting of correct email- 
addresses out of a $t x t$-file, a coding/decoding of a TEXT file, useful restructurings of the TEXT files, and many others.

The Maple package of releases 6, 7 and 8 have a number of means for creation of mechanisms of operation with the user libraries (structurally analogous to the main Maple library) of procedures and modules, allowing in the Maple environment to use them at a level of access, analogous for built-in means of the package. The procedures, represented by the eleventh group, allow to substantially simplify operation with user libraries. So, the basic procedure User_pflM ensures making and update of user's libraries structurally analogous to main Maple library, with their subsequent logical linking with the main library, what ensures access to functional means, contained in them, at a level of the built-in package means. At that, at the procedure call it is enough to indicate only a set or a list of names of the saved means and a name of sub-directory for the created library. Along with that, this group contains a number of means for support of the simplest ways of creation of the user procedures libraries and organization of access to them. User libraries, created in such a way, have the organization, more simple, than the main Maple library. In a series of cases such libraries are the more simple in use.

For interactive making or updating of Help database of the user's library the Maple gives means of the Help-group of the Graphical User Interface (GUI), namely: two functions 'Save to Database" and 'Remove Topic'. However, in view of a series of differences of releases 6,7 and 8 the helpman procedure providing automatic up-date of the user's Help database on the basis of the beforehand prepared $m w s$-files had been created. At that, it is necessary to note one important circumstance. In some cases by a chain of functions "Help -> Save to Database" of the Maple's GUI, a putting into user Help database of a Mapl-worksheet is not guaranteed (at registering it in the corresponding index file Maple.ind), whereas the procedure helpman is free of this defect.

Having created own library of procedures with use of the above procedure User_pflM, or in a different way, quite naturally arises a problem of its optimization, in particular, with the purpose of a frequency revealing of use of means, contained in it, and basic computer resources used by them. In this context the problem of optimization of the user libraries is a rather important. For these purposes a rather useful appears the procedure StatLib(L), providing gathering of the basic statistics on the indicated L library and the return it for the subsequent analysis. Along with that, the group contains means for support of various useful procedures for operation with the user libraries of different organization, including means for restoration of the damaged libraries.

At last, means of groups 12-14 support a series of useful enough procedures for problems solving in analysis, linear algebra and simple statistics. In particular, these means provide a series of very useful procedures for operation with rtable-objects, for special sorting of numeric objects of types \{array, matrix, Matrix\}, for construction of linear and quadratic regression models, for hypotheses testing and analysis of time series. The detailed description of all means composing the above library is represented in our monograph [4]. The means, represented by the given library, relieved a lot of the questions which have arisen in discussions of members of the Maple User Group and outlined a series of interesting ways of subsequent development of the library. 


\section{Structural Library Organization}

The library is structurally similar to the main Maple library, is supplied with the advanced Help-system about means located in it and is logically linked to the main Maple library, providing access to means contained in it similarly to the package means. For the library no more than $6 \mathrm{MB}$ of free space on hard disk is demanded. The library is located in separate subdirectory of LIB-directory with the main Maple library. The library subdirectory contains three files Maple.hdb, Maple.ind, Maple.lib (analogously to the main Maple library), and also special file Maple_U.cmd which contains all history of updating of the library since its creating.

The simple guide describes installation of library at presence on the personal computer of the installed Maple package of releases 6, 7 and/or 8. The library admits two levels of installation, namely: (1) implantation into a Maple environment of the fully-prepared library of the corresponding release $(6,7$ or 8$)$ with posterior linking of it with the main Maple library, and (2) execution in Maple environment of a special $m w s$-file with definitions of the library members with posterior adding of Help database into the library installed in this way.

The library Help system gives the detailed enough description of all means contained in the library with the most typical examples of their use, including analysis of erroneous and especial situations, which can arise at their execution. In one's turn, the monograph [4] represents the detailed description of the software contained in the library, including source texts of the procedures.

The library delivery includes the source texts of all procedures and modules. That allows not only easily to immerse them into the Maple environment of releases 6, 7 and 8 on many computer platforms, but also to use as an useful enough illustrative material at mastering of the advanced programming in the package environment. Many of the software, represented here, use useful enough methods in the practical respect and also receptions of programming (including and non-standard ones) in the package environment, conditioning to them as the applied, and educational interest.

The library is designed for the broad audience of experts, teachers, post-graduates and students of the natural-science professions which use in own professional work the Maple package of releases 6, 7 or 8 on Windows platform. The library is delivered as shareware software on the basis of separate request by email indicated above; for the more detailed acquaintance with the library its demo can be requested.

\section{References}

1. Aladjev, V.Z., Bogdevicius, M.A.: Interactive Maple: Solution of Mathematical, Statistical and Engineering-Physical Problems. International Academy of Noosphere, Tallinn-Vilnius (2001)

2. Aladjev, V.Z.: Effective Work in Maple 6/7. Laboratory of Basic Knowledge, Moscow (2002) + CD-ROM, in Russian

3. Aladjev, V.Z., Bogdevicius, M.A., Prentkovskis, O.V.: New Software for Mathematical Package Maple of Releases 6, 7 and 8. Vilnius Technical University, Vilnius (2002)

4. Aladjev, V.Z., Vaganov, V.A. Computer Algebra System Maple: A New Software Library. International Academy of Noosphere, Tallinn (2002) 